\title{
Claus Offe \\ Bemerkungen zur spieltheoretischen Neufassung des Klassenbegriffs bei Wright und Elster
}

Es ist ein heute verbreitet empfundenes und bekundetes Ärgernis, daß man für die Zwecke der sozialökonomischen und soziopolitischen Analyse industriell entwickelter Gesellschaften mit dem Klassenbegriff weniger ausrichten kann, als man erwarten möchte, wenn man sich an den großen Vorbildern der Marxschen und der marxistischen Tradition orientiert. Die Enttäuschung und Frustration, die sich überall dort rasch einstellt, wo der Klassenbegriff nicht nur locker und deklamatorisch zur Konsolidierung des eigenen politischen Weltbildes, sondern als eine zentrale sozialwissenschaftliche Kategorie verwendet werden soll, erklärt sich aus dem tatsächlich gewaltigen Anspruch, der im klassischen Marxismus mit diesem Konzept verbunden war. Dabei besteht die heute so schwer einzulösende Ambition des Klassenbegriffes vor allem darin, daß mit ihm zugleich die sozialökonomische Struktur einer Gesellschaft und das typische, »rationelle« soziale Handeln der Mitglieder dieser Gesellschaft erfaßt werden sollte; und andererseits darin, daß simultan die "Statik (d.h. die identische Reproduktion) einer Sozialstruktur und ihre "Dynamik« (d.h. die in sozialen Konflikten durchgesetzte institutionelle Umformung der Sozialstruktur) erklärlich gemacht werden sollte. Kurz: soziale Klassen sind kollektive Strukturlagen sozialer Akteure, und diese Strukturlagen konditionieren und programmieren bestimmte Arten sozialen Handelns, und zwar sowohl dasjenige soziale Handeln, das (bis zu bestimmten Grenzen) zur Bestätigung und identischen Reproduktion der Ausgangsstruktur führt, wie dasjenige soziale Handeln, das (jenseits jener Grenze) zur Überwindung und Neukonstitution der institutionellen Kernstruktur einer Gesellschaft führt.

Vergegenwärtigen wir uns kurz die beiden Handlungskreise, den »reproduktiven« und den "transformativen «, die sich, dem klassentheoretischen Denkmuster zufolge, an die Ausgangsstruktur der Produktionsverhältnisse anschließen und mit einer gewissen Z $Z_{\text {wangsläufigkeit }}$ aus ihnen hervorgehen sollen. Zuerst zum reproduktiven Aspekt dieses Zusammenhanges von Struktur und Handeln, der verdeutlichen soll, wie und weshalb eine kapitalistische Ausgangsstruktur (definiert durch privates Eigentum an Produktionsmitteln und freie Lohnarbeit) zu typischen Handlungen führt, die jene Ausgangsstruktur reproduzieren, ausweiten und befestigen. Jene reproduktive Feedback-Schleife setzt sich aus den folgenden Handlungsstrategien der beteiligten Akteure zusammen: (a) die miteinander konkurrierenden Kapitaleigentümer versuchen, um sich als kapitalistisch fungierende Unternehmer im Markt halten zu können, die Arbeitskraft so effizient wie möglich zu nutzen und so niedrig wie möglich zu entlohnen. Sie verdrängen und enteignen dadurch konkurrierende, weniger effiziente Eigentümer und füllen so indirekt das Reservoir der Lohnarbeit auf, dessen Marktmacht mithin relativ zur Marktmacht der Arbeitskraft-Nachfrager sinkt. Da (b) der eigentumslose Teil der Erwerbsbevölkerung keine andere Option hat, als die eigene Arbeitskraft zu marktbestimmten Bedingungen zum Verkauf anzubieten, wächst durch ihren produktiven Einsatz die Ausbeutungsrate, d.h. derjenige Anteil des kollektiven Wertprodukts, der als Mehrwert an die Eigentümer der Produktionsmittel übergeht und von ihnen in der nächsten Runde erneut als 
Kapital eingesetzt wird. So führt von Runde zu Runde eine bestimmte Ausgangsstruktur zu einem typisch-zwangsläufigen Handeln der beteiligten Akteure (d.h. zu Produktivitätssteigerung bei den kapitalistischen Eigentümern und zu produktiver und mehrwert-ergiebiger Arbeit bei den eigentumslosen Arbeitskräften) was wiederum zur erweiterten Reproduktion der Ausgangsbedingungen führt, auf deren Grundlage der Prozeß dann erneut einsetzt. Die Struktur selbst ist es, welche die Eigentümer zum Rationalisieren und die Lohnarbeiter zum Arbeiten zwingt, und dies wiederum führt zur Befestigung und Ausbreitung der Struktur selbst, nämlich zur Akkumulation von Kapital und zur Proletarisierung der Lohnarbeit. Dieser reproduktiven Schleife steht jedoch - und darin besteht für jedes kritische und revolutionäre Denken die Pointe der Klassentheorie - eine transformative Schleife gegenüber, die sich mit der Sequenz: Ausgangsstruktur - kollektives Handeln - Strukturveränderung schematisch darstellen läßt. Das bedeutet, daß die Struktur nicht nur struktur-konsistentes Handeln, sondern auch (auf individueller und schließlich kollektiver Ebene) konflikthaftes und die Struktur selbst im Ergebnis desintegrierendes Handeln hervorruft. Die Arbeiter arbeiten nicht nur, sondern sie organisieren sich auch zum (schließlich) erfolgreichen Widerstand gegen den ihnen auferlegten strukturellen Verkaufszwang für ihre Arbeitskraft. Und die Kapitalisten rationalisieren nicht nur den Akkumulationsprozeß, sondern sie desorganisieren und irrationalisieren ihn gleichzeitig, indem sie (wie in dem Theorem der tendenziell fallenden Profitrate oder auch in Unterkonsumptionstheorien behauptet) in der Blindheit ihres einzelwirtschaftlichen Profitstrebens die kollektiven Bedingungen zukünftiger Akkumulation untergraben.

Die beiden zentralen Behauptungen jeder Klassentheorie, mit deren Beweisbarkeit diese steht und fällt, lassen sich deswegen folgendermaßen formulieren: (1) Alles für den Bestand und die Transformation einer historischen Gesellschaftsformation relevante soziale Handeln ist durch die Produktionsverhältnisse, d.h. durch die (kapitalistische) Verteilung von Verfügungsrechten über Produktivkräfte konditioniert, und (2) alles so konditionierte Handeln hat funktional ambivalente, d.h. sowohl reproduktive wie transformative Konsequenzen. Worin bestehen nun die Schwierigkeiten, auf die man allenthalben bei dem Versuch stößt, dieses ungemein elegante, geschlossene und schon deshalb sozusagen »denkästhetisch « faszinierende Analyseschema anzuwenden und einzulösen?

Was die »reproduktive « Schleife betrifft, so ist zunächst umstritten, ob die laufende identische Selbsterneuereung des sozialökonomischen Systems tatsächlich durch die Mechanismen der Ausbeutung mehrwert-produktiver Lohnarbeit und die strukturellen Verkaufszwänge für Arbeitskraft erklärt werden kann. Die entsprechenden Zweifel knüpfen sich an zwei Beobachtungen. Auf der einen Seite besteht zwar der »Verkaufszwang « für die eigentumslose Arbeitskraft in (wohlfahrtsstaatlich mehr oder weniger abgepufferter Weise) fort, aber für einen wachsenden Teil der so in den Kapitalprozeß hineingezogenen gesellschaftlichen Arbeitskraft, nämlich für die »neue Mittelklasse«, ist durchaus bestreitbar, ob diese Arbeit auch zugleich Mebrwert bildet und damit zur Reproduktion des Kapitals beiträgt. Das jedenfalls wäre eine für viele verwaltende, kommerzielle, d.h. die (im privaten und öffentlichen Sektor) dienstleistende Arbeiten schwer einlösbare Behauptung, da die entsprechenden (meist immateriellen) Arbeits"produkte" eher mehrwert-absorbierenden als mehrwert-erzeugenden Charakter haben. Zweitens kommt hinzu, daß nicht nur (ein Teil der) Arbeit keine wertbildende Funktion hat, sondern daß außerdem ein Teil der Wertbildung nicht durch Lohnarbeit, zumindest nicht in direkter Proportion zu dem Volumen der verausgabten Lohnarbeit stattfindet. Dieser zweite Bruch in der »reproduktiven« Schleife bezieht sich darauf, daß 
Technik und Wissenschaft, wie verschiedentlich vermutet worden ist, zu einer selbständigen (wenn nicht gar »ersten «) Wertquelle geworden sind. Zusammengenommen lenken diese beiden Phänomene - das der nicht-wertschaffenden Dienstleistungsarbeit und das der zu groBen Teilen nicht unmittelbar dem Kapital subsumierten Arbeit der wissenschaftlich-technischen Intelligenz - den Blick auf das Problem der neuen Mittelklasse.

Diesem Kontext von Problemen möchte Wright durch seine Ausweitung und innere Differenzierung des Ausbeutungsbegriffs gerecht werden, die er im Anschluß an die außerordentlich einflußreichen Arbeiten von John Roemer vornimmt. Bei dieser begrifflichen Prozedur sind zwei Schritte zu unterscheiden. Es handelt sich einerseits um die Behauptung, daß Ausbeutung keineswegs nur, wie bisher meist angenommen, innerbalb der fabrikförmig-organisierten Beziehung zwischen Lohnarbeit und Kapital vonstatten gehen könne. Vielmehr sei von Ausbeutung überall dort zu sprechen, wo »die einen « deshalb mehr Einkommen erzielen, weil »die anderen « weniger Einkommen erzielen. Ausbeutung bezeichnet hier die Struktur eines Spieles, in der die Gewinne der einen Seite kausal auf die Verluste der anderen Seite zurïckgeführt werden können. Hier drängt sich der Einwand auf, ob durch eine solche begriffliche Ausdehnung des Ausbeutungsbegriffes dieser nicht völlig konturlos und unscharf wird? Nein, antwortet Roemer (oder jedenfalls Wright), denn wir haben das folgende Kriterium dafür, ob eine Verteilungssituation auf Ausbeutung beruht: es muß in der gegebenen Lage eine alternative Verteilungsstruktur wirklich vorstellbar ("hypothetically feasiblea) sein, in der die Schlechtergestellten bessergestellt werden und deswegen zugleich die bisher Bessergestellten relativ verlieren. Aber dadurch ist das Problem nur auf die Frage verschoben, was hier »wirklich vorstellbar « bedeutet? Eine Ausbeutungsbeziehung würde dann davon abhängig gemacht, daß die von ihr Betroffenen sie als solche definieren, - z.B., indem sie anfangen, von einem Robin Hood zu träumen, der den Reichen ihren Reichtum wegnehmen und den Armen zur Verfügung stellen könnte, obwobl vielleicht der Reichtum der Reichen gar nicht kausal auf einem Ausbeutungsverhältnis gegenüber den Armen beruht. Damit wird offensichtlich der Ausbeutungsbegriff der beliebigen Deutung und dem subjektiven Für-MöglichHalten anheimgestellt, und es ist keine ungleiche Verteilungssituation mehr denkbar, die nicht gleichzeitig Ausbeutung wäre. Der Zusammenhang wird, so scheint es, auf den Kopf gestellt: Die Vorstellung von einer nicht-ausbeuterischen Gesellschaft ergibt sich nicht mehr aus der Erfahrung der (tatsächlichen) Ausbeutung, sondern umgekehrt ergibt sich die tatsächliche Ausbeutung als Schlußfolgerung aus jener Vorstellung. Die Kausalität, die zwischen Armut und Reichtum hergestellt wird, ist bloßes Derivat einer vorgestellten anderen Kausalität und ihrer (einstweiligen) Unwirklichkeit.

Mit dieser radikalen Ausweitung und Subjektivierung des Ausbeutungsbegriffes handelt man sich noch eine weitere Schwierigkeit ein. Wenn man sich der von John Roemer eingeführten Begrifflichkeit anschließt, dann wird weniger selbstverständlich, als es auf den ersten Blick erscheint, ob einem ausbeuterischen Verteilungssystem ein nicht-ausbeuterisches tatsächlich vorgezogen werden soll. Es wäre nämlich denkbar, daß das als Alternative imaginierte ("hypothetically feasible«) Verteilungsmuster zwar nicht ausbeuterisch, d.h. egalitär, dafür aber auch weniger produktiv wäre. Niemand hätte weniger als irgendjemand anders, aber alle hätten weniger als bisher. Dieser Zusammenhang zwischen Verteilungsstruktur und Einkommensniveau, der ja die theoretische Trumpfkarte des ökonomischen Liberalismus ist, läßt es als u.U. durchaus zweifelhaft erscheinen, ob die als ausbeuterisch bloß definierte Situation dem Wunsche der Ausgebeuteten zufolge tatsächlich abgeschafft und in eine egalitäre Struktur überführt werden sollte. So verliert durch die Ausweitung, die der Ausbeutungsbegriff 
durch Roemer erfährt, dieser nicht nur seine analytische Schärfe, sondern auch seine kritische Potenz.

Die Weiterentwicklung dieses Gedankens bei Wright, der ja nicht nur das Problem der neuen Mittelklassen klassentheoretisch bewältigen möchte, sondern auch die Verhältnisse in "real existierenden« sozialistischen Ländern klassentheoretisch in den Griff bekommen will, führt dann zweitens zur Unterscheidung zwischen verschiedenen Mechanismen der Ausbeutung. Zwei von ihnen sind historisch geläufig: Der Ausbeutungsmechanismus, der über das Dispositionsrecht über die Person der Arbeitskraft läuft (Feudalismus), und der Ausbeutungsmechanismus, der über das Dispositionsrecht über die sachlichen Produktionsmittel läuft (Kapitalismus). Zusätzlich zu diesen denkt er an Knappheitsgewinne und Qualifikationsrenten, die sich aus dem Wissen und Können von Experten ergeben, sowie an Ausbeutungsbeziehungen, die sich aus dem Machtstatus innerhalb organisierter Sozialsysteme ergeben. Zweifellos kann man sich leicht vorstellen, daß auch Expertenwissen und organisatorischer Status in modernen Industriegesellschaften die Grundlage dafür bieten, daß sich die Inhaber dieser Privilegien auf Kosten anderer bereichern und diese insofern mausbeuten $\kappa_{3}$ - denn zu jedem dieser Arrangements kann man sich hypothetisch alternative Verhältnisse vorstellen, unter denen (durch Einführung materieller Gleichverteilung bzw. organisatorischer Demokratisierung) jene zusätzlichen Arten der Ausbeutung (die ja nur wegen jener Vorstellbarkeit von Alternativen zu solchen werden!) wieder abgeschafft würden. Es fragt sich jedoch, was man dadurch gewinnt, daß man diese diversen Ausbeutungsverhältnisse und -mechanismen begrifflich verselbständigt, anstatt sie aufeinander zurückzuführen oder jedenfalls in einen funktionalen Zusammenhang zueinander zu stellen. Was man verliert, wenn man dies tut, scheint klar: nämlich jede Rechtfertigung dafür, die Totalität der in "kapitalistischen " Gesellschaftssystemen anzutreffenden Ungleichheiten auf das dort definitionsgemäß dominante Verhältnis zwischen Kapital und Lohnarbeit zurückzuführen. Stattdessen ergibt sich das Bild einer Pluralität von diversen Ausbeutungsbeziehungen, die unverbunden nebeneinander bestehen. Einerseits soll die neue Mittelklasse, sofern sie in Lohnarbeits-Verhältnissen steht, selbst (»kapitalistisch «) ausgebeutet sein, andererseits sollen sich ihre Angehörigen als "Qualifikations-Ausbeuter (»skill-exploiters«) zu Lasten aller anderer (einschließlich des Kapitals?) betätigen. Daß bzw. bis zu welcher Grenze sich in dieser Gemengelage heterogener Ausbeutungsbeziehungen eine speziell kapitalistische Dominante halten sollte (wie Wright immerhin andeutet), bleibt gänzlich undeutlich, weil er seinem Ansatz nach nicht in der Lage ist, die verschiedenen Arten der Ausbeutung, die Kapitalisten gegenüber Lohnarbeitern, Bürokraten gegenüber Mitgliedern, Experten gegenüber Laien (und, so könnte man hinzufügen, Männer gegenüber ihren quasi-feudal kontrollierten Ehefrauen) praktizieren, in eine Rangfolge größerer oder geringerer Zentralität, Dominanz usw. zu bringen.

Dieses polyzentristische Gefüge von Ausbeutungsbeziehungen wird nun von Wright mit dem Argument verteidigt, daß eine so angelegte Klassenanalyse jedenfalls "materialistischer « sei als eine primär an Kategorien der Macht und Herrschaft (»domination «) orientierte Analyse. In der Tat besteht ja die Pointe der Roemerschen Analyse darin, daß die „Despotie der Fabrikarbeit « (Marx) nur als ein Sonderfall von Ausbeutungsbeziehungen dargestellt wird, dem durchaus »herrschaftsfreie« Formen der Ausbeutung gegenübergestellt werden müssen. Mir scheint jedoch das Kapital-Lohnarbeit-Verhältnis gegenüber jenen anderen Ausbeutungsverhältnissen dadurch ausgezeichnet zu sein, daß es in zweierlei Hinsicht durch eine komplementäre Rechtsstruktur gesichert und institutionalisiert ist und sich inso- 
fern von jenen Ausbeutungsverhältnissen unterscheidet, die denjenigen zugutekommen, die bloß faktisch am längeren Hebel sitzen und sich günstige Gelegenheiten bzw. kontingente Knappheitsbeziehungen zunutzemachen können. Mit den beiden Hinsichten, in denen das im Verhältnis zwischen Kapital und Arbeit stattfindende Ausbeutungsverhältnis rechtlich und institutionell ausgezeichnet ist, meine ich einerseits jene besondere Befehls- und Organisationsgewalt ( $»$ Direktionsrecht $\ll$ ), die sich als Konnexinstitut des Eigentumsrechts darstellt und innerbetrieblich die organisatorischen Voraussetzungen dafür sichert, daß der Ausbeutungsprozeß überhaupt vonstattengehen kann; andererseits die ebenfalls rechtlich erzeugte Fiktion von Vertragsfreibeit und Waffengleichbeit, die den Arbeitsvertrag umgibt und dadurch seinen Ausbeutungscharakter verhuillt und formalrechtlich dementiert. Beides - sowohl die herrschaftliche Ermächtigung zur Ausbeutung wie ihre rechtliche Verschleierung - sind Besonderheiten der Beziehung zwischen Kapital und Arbeit, für die sich in den anderen der von Wright angeführten Ausbeutungsverhältnisse keine oder doch nur bei weitem schwächere Parallelen finden. Insofern rächt sich die durch das spieltheoretische Paradigma nahegelegte, scheinbar »rein materialistische « Konzentration auf reine Ausbeutungs-im Gegensatz zu Herrschaftsphänomenen darin, daß die Zentralität der Ausbeutungsbeziehung zwischen Lohnarbeit und Kapital, die in der bürgerlichen Gesellschaft zugleich rechtlich privilegiert und rechtlich dementiert wird, aus dem Blick gerät.

Die größte Schwierigkeit, welche die Klassentheorie heute mit der zweiten der oben unterschiedenen beiden Rückkopplungsschleifen, also der »transformativen « Schleife, hat, besteht darin, daß sehr wohl kollektive Akteure die institutionelle Kernstruktur der kapitalistischen Gesellschaft infrage stellen und deren institutionelle Kontinuität bedrohen, daß aber die Identität dieser kollektiven Akteure nicht ohne weiteres klassentheoretisch deduzierbar ist, sondern sich häufig aus anderen, »querliegenden« Bestimmungsgründen ergibt, für die der "proletarische" Klassenstatus der Akteure weder notwendige noch zureichende Bedingung ist. Unter welchen Bedingungen sind kollektive und in ihrem Ergebnis potentiell »transformative Konflikte ihrer sozialen Basis und ihrem sozialstrukturellen Schnittmuster nach Klassenkonflikte, und unter welchen Bedingungen ist dies nicht der Fall? Das ist die Frage, die im Mittelpunkt des Beitrages von Jon Elster steht. Er erwägt einleitend eine rein strukturtheoretische Fassung des Klassenbegriffes, indem er das Kriterium der Verfügung/Nicht-Verfügung über Eigentum, bzw. das Kriterium prüft, ob oder ob nicht ein Akteur objektiv ausgebeutet ist. Diese Kriterien versagen, so Elster, u.a. deswegen, weil zumindest eine erhebliche Zahl von Akteuren nicht genau wissen kann, ob sie gerade ausgebeutet wird, selbst ausbeutet, oder keiner der beiden Gruppen angehört, - und folglich auch nicht, welcher der drei klassenpolitischen Allianzen (Eigentümer, Kleinbürgertum, Proletariat) der einzelne Akteur sich zurechnen muß. Andererseits wird man den Klassenbegriff, wenn man seine strukturtheoretische Komponente nicht aufopfern und damit seine Eigenschaft, eine Brücke zwischen Struktur und Handeln herzustellen, nicht verschenken will, auch nicht allein auf der Handlungsebene festmachen wollen, - etwa nach der Formel: zum Proletariat gehören alle, die sich ihm zugehörig fühlen und entsprechend bandeln. Nach dieser scharfsinnigen Aufdeckung des klassentheoretischen Dilemmas behilft sich Elster allerdings mit einer Notlösung, die nur scheinbar eine Lösung ist: Er definiert soziale Klassen als strukturell induzierte Optimierungsstrategien und ihre Träger. Zu welcher Klasse man gehört, das entscheidet sich an dem Kriterium, was man aufgrund der ökonomischen Umstände, unter denen man lebt und aufgrund der ökonomischen Ressourcen, mit denen man ausgestattet ist, tun muß, um die eigene Lage zu »optimieren«. Hier steht offenbar ein klassenneu- 
traler »homo oeconomicus bei der Definition sozialer Klassen Pate, insofern unterstellt wird, daß alle Akteure »Optimierer« sind. Wohl zutreffend unterstellt er für die Marxsche Klassentheorie, daß Marx kollektives Klassenhandeln als optimale Strategie der Angehörigen der proletarischen Klasse angenommen hat und davon ausgegangen ist, daß sich - im Selbstbildungsprozeß der »Klasse für sich « - jenes kollektive Klassenhandeln auch durchsetzen werde.

Dieser Erwartung gegenüber zeigen sich, so Elster, nun zwei charakteristische und erklärungsbedürftige »abweichende « Phänomene: Einerseits das Vorhandensein kollektiver Akteure, die nicht auf einer homogenen Ausstattung der Mitglieder mit Produktionsmitteln beruhen, und umgekehrt die Situation, daß homogene Strukturlagen nicht zu kollektivem Handeln führen. Es gibt, wie sich zeigt, sehr gute Erklärungen zumindest für das zweite dieser beiden Phänomene, also dafür, daß sich eine gemeinsame Klassenlage nicht mit innerer Zwangsläufigkeit in ein kollektives Klassenhandeln übersetzt. Denn für diesen Übersetzungsprozeß ist, wie wohl Rosa Luxemburg früher und schärfer als alle anderen herausgearbeitet hat, eine Reihe von »katalysatorischen « institutionellen Randbedingungen erforderlich, die diesen Umsetzungsprozeß begünstigen, ermöglichen und fördern.

Ich sehe die zentrale Bedeutung von Elsters klassentheoretischer Analyse darin, daß hier mit zwingenden Argumenten und vorbildlicher Klarheit der Zusammenhang von »objektiver « Klassenlage und kollektivem Klassenhandeln nicht mehr als ein selbstverständlich sich herstellender, sondern als ein politisch-institutionell vermittelter vorgestellt wird. Die Institutionen des kapitalistischen Staates werden in ihrer Eigenschaft durchsichtig gemacht, ein Klassenhandeln der Bourgeoise teils zu erleichtern, teils zu erübrigen, auf der anderen Seite aber ein proletarisches Klassenhandeln zu erschweren und zu desorganisieren. Auch hier stellt sich also, und in Konvergenz mit der oben skizzierten Kritik an dem lupenreichen »Materialismus« von Wright, die zentrale methodische Bedeutung einer "nicht-reduktionistischen politischen Theorie« (Elster) heraus. Über die Wahrscheinlichkeit bzw. Unwahrscheinlichkeit dafür, daß es zu einer Übersetzung von Strukturbedingungen in kollektives Handeln kommt, entscheiden nicht Eigenschaften dieser Strukturbedingungen allein, sondern die institutionalisierten Spielregeln der politischen Verfassung, die, wie Elster hervorhebt, einen eigenständigen Beitrag zur Generierung von Machtpositionen leiste, der nicht seinerseits wieder, will man nicht in die übelsten Gewohnheiten funktionalistischen Denkens zurückfallen, allein als Ergebnis klassenpolitischer Machinationen interpretiert werden kann. Ähnlich wie geltende verfassungspolitische Spielregeln können auch ethnische, religiöse, sprachliche und andere kulturelle Identitäten eine Lösung jenes »Übersetzungsproblems« erleichtern oder erschweren, das zwischen der Ebene der Strukturlagen und der Ebene des sozialen Handelns besteht. Auch hier ist die divide et impera-Interpretation, die kulturelle Identitäten als planvolle klassenpolitische Arrangements (meist zum Zweck der Spaltung der Arbeiterklasse) auffaßt, ein funktionalistischer Kurzschluß. Wenn aber die Spielregeln der politischen Verfassung und die Muster der kulturellen Identitäten, in denen die Akteure stehen, nicht reduktionistisch als Reflexe von Klassenlagen und Klasseninteressen verstanden werden können, wie sind sie, ihre zumindest mrelative Autonomie und ihr dementsprechend autonomer Wandel dann zu verstehen? Über diese Frage schweigt sich Elster, zumindest an dieser Stelle, vollständig aus. Doch wird hier zumindest der gedankliche Schritt deutlich, der im Interesse einer Vervollständigung und Präzisierung der Klassentheorie über deren Grenzen hinaus in Richtung auf die institutionellen und kulturellen Rahmenbedingungen eines kollektiven Klassenhandelns getan werden müßte, wenn die Klassentheorie nicht einer ziemlich öden Praxis ökonomischer Modellrechnungen überlassen werden soll. 